\title{
A systematic review and meta-analysis for effects of exercise interventions on accumulation of subcutaneous, visceral, and ectopic fat in overweight and obese adults: a randomized controlled trial
}

\author{
Junga Lee ${ }^{1, *}$ \\ 1 Sports Medicine and Science, Kyung Hee University, Gyeonggi-do, 17104, Korea \\ * Author to whom correspondence should be addressed.
}

\begin{abstract}
Background: Overweight and obese adults seek effective exercise interventions to reduce accumulated fat, but the effectiveness of these interventions vary across studies. The purpose of this meta-analysis was to investigate the effectiveness of exercise interventions in overweight and obese adults based on measurement of accumulated fat distributions. (2) Methods: Databases were used to select eligible studies for this meta-analysis. Randomized controlled trials with a control and experimental group were included. Degrees of effectiveness of exercise interventions were computed to assess the benefits on reducing weight and subcutaneous, visceral, and ectopic fat accumulation. (3) Results: A total of twenty-one studies were included in this meta-analysis. Participation in exercise interventions showed beneficial effects in reducing weight and subcutaneous and visceral fat. The effectiveness of exercise interventions on ectopic fat accumulation could not be assessed due to the limited number of studies measuring ectopic fat. Additionally, effectiveness of exercise interventions that depended on measurements of accumulated fat varied. The average exercise intervention for overweight and obese individuals was moderate to vigorous intensity, 4 times per week, 50 minutes per session, and 22 weeks duration. (4) Conclusions: Participating in exercise interventions has favorable effects on reducing weight and accumulation of subcutaneous and visceral fat.
\end{abstract}

Keywords: fat; obesity; randomized controlled trials; meta-analysis

\section{Introduction}

There are two billion overweight or obese individuals worldwide, and more than $25 \%$ of these are over 18 years old [1]. Obesity in this population rose from about $4.5 \%$ in 1975 to about $13 \%$ in 2016, while the number of those overweight increased from about $22 \%$ to about $39 \%$ [1]. An accumulation of fat is a crucial factor that increases morbidity and mortality. Overweight and obese adults had a higher rate of chronic diseases. A previous meta-analysis reported that being overweight and obese were associated with a higher rate of disease-specific and all-cause mortality [2]. Additionally, ectopic fat, which accumulates in all organs or tissues including skeletal muscle, liver, pericardium, perirenal tissues, and perivascular areas, may also be a cause of chronic disease including cardiorenal metabolic risks [3]. Ectopic fat deposited in skeletal muscle and the liver may influence systemic metabolic energy, and pericardial fat is associated with coronary atherosclerosis [4]. Also, pericardial, perivascular, and renal sinus fat may affect adjacent anatomic organs leading to direct lipotoxicity resulting in cytokine secretion [5,6]. Decreasing accumulation of fat is preventive healthy behavior.

Participation in exercise or dietary interventions leads to reduced accumulations of fat and improvement in the cardiorenal metabolic risks. A recent meta-analysis among obese children and adolescents demonstrated that exercise or diet had favorable effects on accumulation of fats including ectopic fat [7]. However, the effects of exercise for overweight and obese adults may be different than 
children and adolescents. Also, outcomes of exercise interventions on changes in accumulated fat may be different. This can be ascertained through measuring methods of fat: computed tomography $(\mathrm{CT})$, magnetic resonance imaging (MRI), volume-localized $1 \mathrm{H}$-magnetic resonance spectroscopy (MRS), and dual-energy X-ray absorptiometry (DAX) that identify accumulations of fat in different organs. Also, depending on the different methods, clinically useful measurements of accumulated fat need to be defined. Therefore, the purpose of this meta-analysis was to understand the effects of exercise intervention on overweight and obese adults and to investigate fat distribution differences and ectopic fat measurements.

\section{Materials and Methods}

\subsection{Searching processes}

We followed the Preferred Reporting Items for Systematic Reviews and Meta-analysis statements (PRISMA) [8] and used the MEDLINE and EMBASE databases to identify relevant studies from January 1990 to July 2019 for this meta-analysis. Search terms for eligible articles were accumulated fat; ectopic fat (liver, hepatic, visceral, abdominal, intrahepatic, intramyocellular, myocardial, cardiac, pancreatic); obesity (overweight, adipose tissues); and exercise (aerobic, endurance, strength, resistance). Inclusion criteria were: reporting results of the effects of pre- and post-exercise interventions; recruiting overweight or obesity adults; randomized controlled trials; indicating determination methods for being overweight and obese; and describing measurement technologies including CT, MRI, MRS, and DAX. Exclusion criteria were being a pilot study, systematic review, or meta-analysis review. Missing results for ectopic fat deposits was another exclusion criterion. We also manually searched references cited in review articles to identify further relevant studies. The Cochrane Collaboration's Risk of Bias Tool was used to assess the quality of the selected studies [9].

\subsection{Statistical analysis}

We used Comprehensive Meta-analysis 2nd version software (Biostat, Englewood, USA). The standardized mean difference statistic, which is the difference in treatment and control group means divided by the pooled standard deviation, was used to calculate the effect size. Heterogeneity between study results was tested with the $Q$ test. If $p$-values were less than 0.10 , we considered the results to be homogeneous. Based on the values of I2, we determined inconsistency; $<50 \%$ of I2 for small inconsistency, and $\geq 50 \%$ of I2 for large inconsistency. Risk of bias across studies was assessed by visual inspection of the funnel plot.

\section{Results}

We described the selection process in Figure 1. A total of 21,400 studies from the initial search were found; 21,322 of these were initially excluded due to not being related to our topic of exercise intervention and ectopic fat. Finally, a total twenty-one studies were selected for meta-analysis [10-30]. The basic characteristics of the selected studies including the first author's name, country in which the study was conducted, design of the study, numbers of participants, levels of body mass index (BMI), sex, contents of exercise interventions, and major findings are presented in Table 1. All participants had to be older than 18 years. Exercise types in selected studies consisted of 12 studies using aerobic exercise; 3 studies using resistance exercise; one study using interval exercise; and 5 studies using combined exercise including aerobic exercise such as jogging, walking, and cycling, and resistance exercise such as squats, leg extensions, leg curls, elbow flexions, triceps extensions, lateral pull-downs, bench presses, military presses, lower back extensions, and bent leg sit-ups. The average duration of participating in exercise interventions was 22 weeks, 4 times per week for about 50 minutes. The average number of participants for each exercise intervention was 35 . The average intensity of exercise was from moderate to vigorous intensity exercise. 


\subsection{Effects of exercise interventions on weight}

Overweight and obese individuals who participated in exercise interventions had significantly decreased weight $(\mathrm{d}=-0.61$ [95\% confidence interval, $-0.82-0.41 ; \mathrm{p}=0.00 ; \mathrm{k}=14])$. The degree of effectiveness included all exercise interventions.

\subsection{Effects of exercise interventions on subcutaneous fat as measured with CT and MRI}

Subcutaneous adipose tissues were measured by CT and MRI. Participants engaging in exercise intervention had significantly decreased subcutaneous fat ( $\mathrm{d}=-0.34$ [95\% CI, $-0.55-0.13 ; \mathrm{p}<0.001 ; \mathrm{k}=13]$ ) when the measure of effectiveness included all measurements of subcutaneous fat, including those obtained from CT and MRI. Subgroup analysis of CT measurements showed a significant decrease in subcutaneous fat $(\mathrm{d}=-0.30$ [95\% CI, $-0.55-0.04 ; \mathrm{p}=0.02 ; \mathrm{k}=8]$ ]), but MRI measurements $(\mathrm{d}=-0.05$ [95\% CI, $-0.78-0.68 ; \mathrm{P}=0.90 ; \mathrm{k}=4])$ did not show a significant decrease in subcutaneous fat.

\subsection{Effects of exercise interventions on visceral fat as measured from CT and MRI}

Visceral fat was measured by $\mathrm{CT}$ and MRI. Exercise interventions were effective in reducing visceral fat ( $\mathrm{d}=-0.65$ [95\% CI, $-0.90-0.40 ; \mathrm{p}=0.001 ; \mathrm{k}=11])$. Subgroup analysis demonstrated decreased visceral fat measured by both CT ( $\mathrm{d}=-0.83$ [95\% CI, -1.18-0.48; $\mathrm{p}=0.001 ; \mathrm{k}=14])$, and MRI ( $\mathrm{d}=-0.43$ [95\% $\mathrm{CI},-0.80-0.05 ; \mathrm{p}=0.001 ; \mathrm{k}=4]$ ). This section may be divided by subheadings. It should provide a concise and precise description of the experimental results, their interpretation as well as the experimental conclusions that can be drawn.

\subsection{Figures, and Tables}

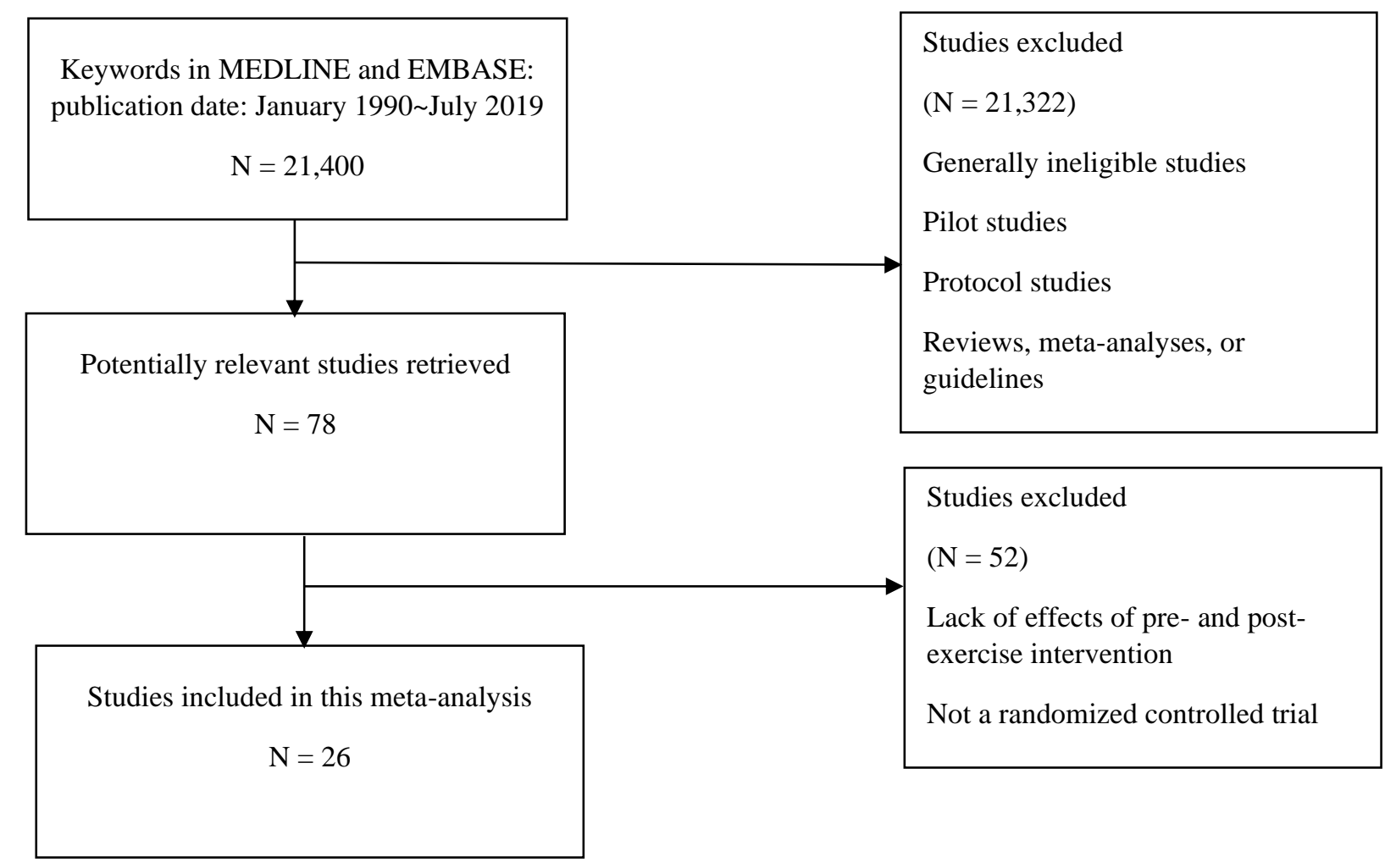

Figure 1. Selection process for the systematic review and meta-analysis 
Studyname

\begin{tabular}{|c|c|c|c|c|c|c|c|}
\hline & $\begin{array}{c}\text { Std diff } \\
\text { in means }\end{array}$ & $\begin{array}{l}\text { Standard } \\
\text { error }\end{array}$ & Variance & $\begin{array}{c}\text { Lower } \\
\text { limit }\end{array}$ & $\begin{array}{c}\text { Upper } \\
\text { limit }\end{array}$ & Z-Value & $p$-Value \\
\hline Ross (2004) weight loss & -0.83 & 0.31 & 0.09 & -1.43 & -0.23 & -2.71 & 0.01 \\
\hline Ross (2004) no weight loss & -0.11 & 0.28 & 0.08 & -0.66 & 0.44 & -0.40 & 0.69 \\
\hline Park (2003) aerobic exercise & -2.92 & 0.64 & 0.41 & -4.18 & -1.66 & -4.54 & 0.00 \\
\hline Park (2015) combined & -1.52 & 0.51 & 0.26 & -2.52 & -0.53 & -3.00 & 0.00 \\
\hline Inving (2008) low & 0.39 & 0.49 & 0.24 & -0.57 & 1.34 & 0.79 & 0.43 \\
\hline Inving (2008) high & 0.09 & 0.45 & 0.20 & -0.78 & 0.96 & 0.20 & 0.84 \\
\hline Hunter (2010) aerobic & -0.75 & 0.33 & 0.11 & -1.40 & -0.09 & -2.23 & 0.03 \\
\hline Hunter (2010) resistnace & -0.19 & 0.31 & 0.09 & -0.80 & 0.41 & -0.63 & 0.53 \\
\hline Johnson (2009) & -0.91 & 0.50 & 0.25 & -1.89 & 0.06 & -1.83 & 0.07 \\
\hline Larson-Meyer (2006) & -2.86 & 0.59 & 0.35 & -4.02 & -1.69 & -4.82 & 0.00 \\
\hline Ibanez (2010) & -0.53 & 0.44 & 0.19 & -1.39 & 0.33 & -1.20 & 0.23 \\
\hline Idoate (2011) & -0.03 & 0.40 & 0.16 & -0.82 & 0.75 & -0.08 & 0.93 \\
\hline Batrakoulis (2018) 20weeks & -0.70 & 0.36 & 0.13 & -1.40 & -0.01 & -1.98 & 0.05 \\
\hline \multirow[t]{2}{*}{ Batrakoulis (2018) 40weeks } & -0.81 & 0.36 & 0.13 & -1.51 & -0.10 & -2.25 & 0.02 \\
\hline & -0.61 & 0.10 & 0.01 & -0.82 & -0.41 & -5.88 & 0.00 \\
\hline
\end{tabular}

Test for heterogeneity: $\left(\mathrm{Q}=45.62, \mathrm{P}<0.001, \mathrm{I}^{2}=71.50 \%\right)$
Statistics for each study

Weight

\begin{tabular}{|c|c|c|c|c|c|c|c|}
\hline \multirow[t]{2}{*}{ Study name } & \multicolumn{7}{|c|}{ Statistics for each study } \\
\hline & $\begin{array}{l}\text { Std diff } \\
\text { in means }\end{array}$ & $\begin{array}{c}\text { Standard } \\
\text { error }\end{array}$ & \multicolumn{3}{|c|}{ Lower Upper } & \multicolumn{2}{|c|}{ Z-Value $p$-Value } \\
\hline & & & & & & & \\
\hline U3) aeroorce & -0.21 & 0.45 & $0 . \angle 0$ & -1.15 & 0.6 & -0. & \\
\hline Park (2015) combined & -0.20 & 0.45 & 0.20 & -1.07 & 0.68 & -0.44 & \\
\hline Hunter (2010) aerobic deep & -0.27 & 0.33 & 0.11 & -0.90 & 0.37 & -0.82 & \\
\hline Hunter (2010) resistance deep & -0.54 & 0.31 & 0.10 & -1.16 & 0.07 & -1.74 & \\
\hline Hunter (2010) aerobic superfical & -0.39 & 0.33 & 0.11 & -1.03 & 0.25 & -1.20 & \\
\hline Hunter (2010) resistance superfical & -0.27 & 0.31 & 0.09 & -0.87 & 0.33 & -0.88 & \\
\hline Irving (2008) low intensity & -0.04 & 0.48 & 0.23 & -0.99 & 0.91 & -0.09 & \\
\hline Irving (2008) high intensity & -0.10 & 0.45 & 0.20 & -0.97 & 0.78 & -0.22 & 0.8 \\
\hline Ross (2004) & -0.57 & 0.30 & 0.09 & -1.16 & 0.02 & -1.88 & \\
\hline Johnson (2009) & 1.19 & 0.51 & 0.26 & 0.19 & 2.20 & 2.32 & \\
\hline banez (2010) & 0.04 & 0.40 & 0.16 & -0.74 & 0.83 & 0.11 & \\
\hline Larson-Meyer (2006) & -2.39 & 0.55 & 0.30 & -3.46 & -1.32 & -4.37 & \\
\hline Idoate (2011) & -0.63 & 0.44 & 0.20 & -1.50 & 0.24 & -1.41 & \\
\hline & -0.34 & 0.11 & 0.01 & -0.55 & -0.13 & -3.22 & \\
\hline
\end{tabular}

\footnotetext{
Test for heterogeneity: $\left(\mathrm{Q}=26.23, \mathrm{P}<0.001, \mathrm{I}^{2}=54.25 \%\right)$
}

Subcutaneous fat: all measurements

\begin{tabular}{|c|c|c|c|c|c|c|c|}
\hline \multirow[t]{2}{*}{ Study name } & \multicolumn{7}{|c|}{ Statistics for each study } \\
\hline & $\begin{array}{l}\text { Std diff } \\
\text { in means }\end{array}$ & $\begin{array}{l}\text { Standard } \\
\text { error }\end{array}$ & Variance & $\begin{array}{l}\text { Lower } \\
\text { limit }\end{array}$ & $\begin{array}{l}\text { Upper } \\
\text { limit }\end{array}$ & Z-Value $p$ & -Vali \\
\hline Park (2003) aerobic exercise & -0.27 & 0.45 & 0.20 & -1.15 & 0.61 & -0.61 & \\
\hline Park (2015) conbined & -0.20 & 0.45 & 0.20 & -1.07 & 0.68 & -0.44 & \\
\hline Hunter (2010) aerobic deep & -0.27 & 0.33 & 0.11 & -0.90 & 0.37 & -0.82 & \\
\hline Hunter (2010) resistance deep & -0.54 & 0.31 & 0.10 & -1.16 & 0.07 & -1.74 & \\
\hline Hunter (2010) aerobic superfical & -0.39 & 0.33 & 0.11 & -1.03 & 0.25 & -1.20 & \\
\hline Hunter (2010) resistance superfical & -0.27 & 0.31 & 0.09 & -0.87 & 0.33 & -0.88 & \\
\hline Irving (2008) low intensity & -0.04 & 0.48 & 0.23 & -0.99 & 0.91 & -0.09 & \\
\hline \multirow[t]{2}{*}{ Irving (2008) high intensity } & -0.10 & 0.45 & 0.20 & -0.97 & 0.78 & -0.22 & \\
\hline & -0.30 & 0.13 & 0.02 & -0.55 & -0.04 & -2.29 & \\
\hline
\end{tabular}

Test for heterogeneity: $\left(\mathrm{Q}=1.26, \mathrm{P}=0.02, \mathrm{I}^{2}=00 \%\right)$
Std diff in means and $95 \% \mathrm{Cl}$

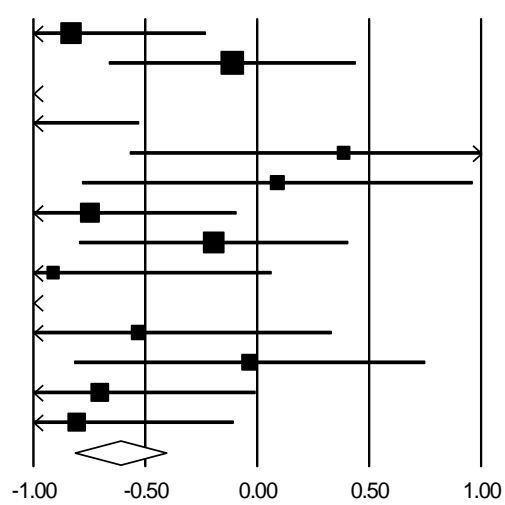

Std diff in means and $95 \% \mathrm{C}$

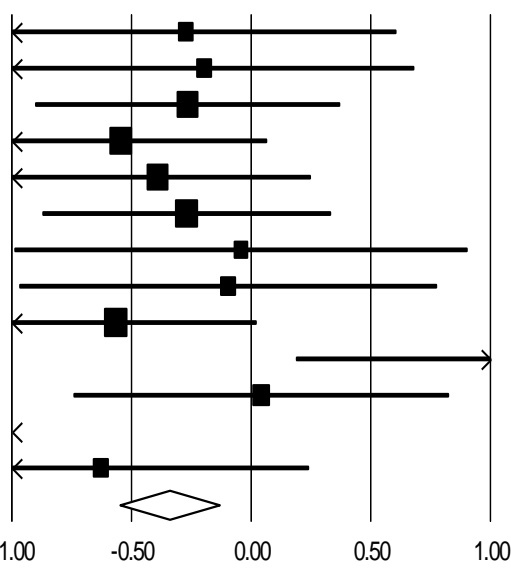

Subcutaneous fat: CT

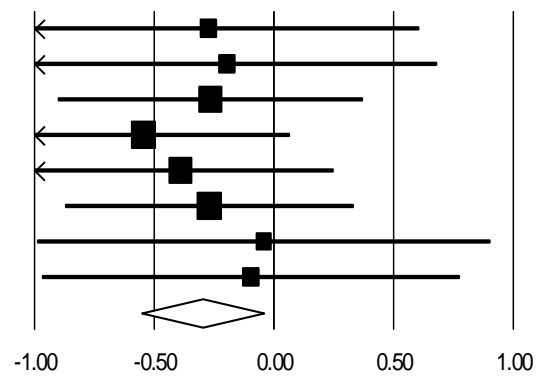

Std diff in means and $95 \% \mathrm{C}$ 


\begin{tabular}{lrrrrrrrr} 
Study name & \multicolumn{8}{c}{ Statistics for each study } \\
\cline { 5 - 8 } & $\begin{array}{c}\text { Std diff } \\
\text { in means }\end{array}$ & $\begin{array}{c}\text { Standard } \\
\text { error }\end{array}$ & $\begin{array}{c}\text { Variance } \\
\text { L }\end{array}$ & $\begin{array}{c}\text { Lower } \\
\text { limit }\end{array}$ & $\begin{array}{c}\text { Upper } \\
\text { limit }\end{array}$ & Z-Value & $p$-Value \\
Ross (2004) & -0.57 & 0.30 & 0.09 & -1.16 & 0.02 & -1.88 & 0.06 \\
Johnson (2009) & 1.19 & 0.51 & 0.26 & 0.19 & 2.20 & 2.32 & 0.02 \\
Ibanez (2010) & 0.04 & 0.40 & 0.16 & -0.74 & 0.83 & 0.11 & 0.92 \\
Idoate (2011) & -0.63 & 0.44 & 0.20 & -1.50 & 0.24 & -1.41 & 0.16 \\
& -0.18 & 0.20 & 0.04 & -0.56 & 0.21 & -0.91 & 0.36
\end{tabular}

Test for heterogeneity: $\left(\mathrm{Q}=10.13, \mathrm{P}=0.90, \mathrm{I}^{2}=70.37 \%\right)$

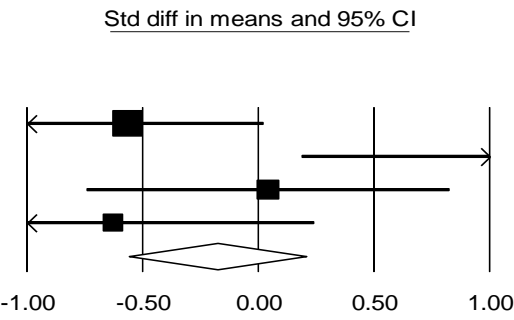

Subcutaneous fat: MRI

Studyname

Park (2003) aerobic exercise
Park (2015) combined
Hunter (2010) aerobic
Hunter (2010) resistance
Ining (2008) low intensity
Ining (2008) high intensity
Ross (2004)
Johnson (2009)
Ibanez (2010)
Larson-Meyer (2006)
Idoate (2011)

\begin{tabular}{rrrrrr}
\multicolumn{7}{c}{$\begin{array}{c}\text { Statistics for each study } \\
\text { Std diff } \\
\text { in means }\end{array}$} & $\begin{array}{c}\text { Standard } \\
\text { error }\end{array}$ & $\begin{array}{c}\text { Lower } \\
\text { Variance }\end{array}$ & $\begin{array}{c}\text { Upper } \\
\text { limit }\end{array}$ & $\begin{array}{c}\text { limit } \\
\text { Z-Value }\end{array}$ \\
-5.83 & 1.02 & 1.05 & -7.84 & -3.82 & -5.69 \\
-4.86 & 0.89 & 0.79 & -6.60 & -3.12 & -5.47 \\
-0.78 & 0.34 & 0.11 & -1.44 & -0.12 & -2.33 \\
-0.54 & 0.31 & 0.10 & -1.15 & 0.07 & -1.73 \\
-0.16 & 0.48 & 0.23 & -1.11 & 0.79 & -0.33 \\
-0.11 & 0.45 & 0.20 & -0.99 & 0.76 & -0.25 \\
-0.74 & 0.30 & 0.09 & -1.34 & -0.15 & -2.44 \\
-0.73 & 0.49 & 0.24 & -1.69 & 0.24 & -1.48 \\
-0.09 & 0.40 & 0.16 & -0.87 & 0.70 & -0.22 \\
-0.75 & 0.43 & 0.19 & -1.60 & 0.10 & -1.74 \\
-0.03 & 0.40 & 0.16 & -0.81 & 0.76 & -0.07 \\
-0.65 & 0.13 & 0.02 & -0.90 & -0.40 & -5.17
\end{tabular}

Test for heterogeneity: $\left(\mathrm{Q}=55.31, \mathrm{P}<0.001, \mathrm{I}^{2}=81.92 \%\right)$

$\begin{array}{lr}\text { Value } & p \text {-Value } \\ -5.69 & 0.00 \\ -5.47 & 0.00 \\ -2.33 & 0.02 \\ -1.73 & 0.08 \\ -0.33 & 0.74 \\ -0.25 & 0.80 \\ -2.44 & 0.01 \\ -1.48 & 0.14 \\ -0.22 & 0.82 \\ -1.74 & 0.08 \\ -0.07 & 0.95 \\ -5.17 & 0.00\end{array}$

0.00

Visceral fat: all measurements

Studyname

\begin{tabular}{|c|c|c|c|c|c|c|}
\hline \multirow[b]{2}{*}{$\begin{array}{l}\text { Std diff } \\
\text { in means }\end{array}$} & \multicolumn{6}{|c|}{ Statistics for each study } \\
\hline & $\begin{array}{l}\text { Standard } \\
\text { error }\end{array}$ & Variance & $\begin{array}{c}\text { Lower } \\
\text { limit }\end{array}$ & $\begin{array}{c}\text { Upper } \\
\text { limit }\end{array}$ & Z-Value & $p$-Value \\
\hline-5.83 & 1.02 & 1.05 & -7.84 & -3.82 & -5.69 & 0.00 \\
\hline-4.86 & 0.89 & 0.79 & -6.60 & -3.12 & -5.47 & 0.00 \\
\hline-0.78 & 0.34 & 0.11 & -1.44 & -0.12 & -2.33 & 0.02 \\
\hline-0.54 & 0.31 & 0.10 & -1.15 & 0.07 & -1.73 & 0.08 \\
\hline-0.16 & 0.48 & 0.23 & -1.11 & 0.79 & -0.33 & 0.74 \\
\hline-0.11 & 0.45 & 0.20 & -0.99 & 0.76 & -0.25 & 0.80 \\
\hline-0.83 & 0.18 & 0.03 & -1.18 & -0.48 & -4.59 & 0.00 \\
\hline
\end{tabular}

Test for heterogeneity: $\left(\mathrm{Q}=49.77, \mathrm{P}<0.001, \mathrm{I}^{2}=89.95 \%\right)$

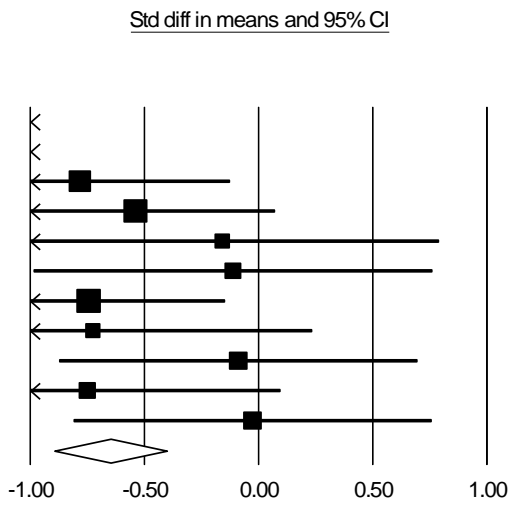

Std diff in means and $95 \% \mathrm{Cl}$

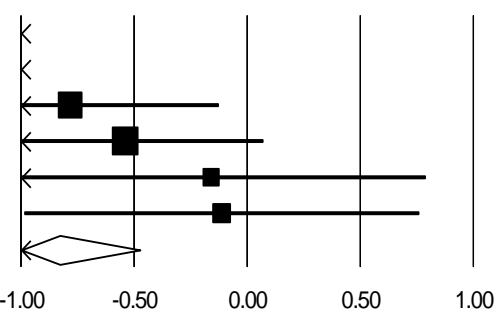

Visceral fat: CT

\begin{tabular}{|c|c|c|c|c|c|c|c|}
\hline \multirow[t]{2}{*}{ Study name } & \multicolumn{7}{|c|}{ Statistics for each study } \\
\hline & $\begin{array}{l}\text { Std diff } \\
\text { in means }\end{array}$ & $\begin{array}{l}\text { Standard } \\
\text { error }\end{array}$ & Variance & $\begin{array}{c}\text { Lower } \\
\text { limit }\end{array}$ & $\begin{array}{l}\text { Upper } \\
\text { limit }\end{array}$ & Z-Value & $p$-Value \\
\hline Ross (2004) & -0.74 & 0.30 & 0.09 & -1.34 & -0.15 & -2.44 & 0.01 \\
\hline Johnson (2009) & -0.73 & 0.49 & 0.24 & -1.69 & 0.24 & -1.48 & 0.14 \\
\hline lbanez (2010) & -0.09 & 0.40 & 0.16 & -0.87 & 0.70 & -0.22 & 0.82 \\
\hline Idoate (2011) & -0.03 & 0.40 & 0.16 & -0.81 & 0.76 & -0.07 & 0.95 \\
\hline & -0.43 & 0.19 & 0.04 & -0.80 & -0.05 & -2.24 & 0.02 \\
\hline
\end{tabular}

Test for heterogeneity: $\left(\mathrm{Q}=3.17, \mathrm{P}=0.37, \mathrm{I}^{2}=5.28 \%\right)$
Std diff in means and $95 \% \mathrm{Cl}$

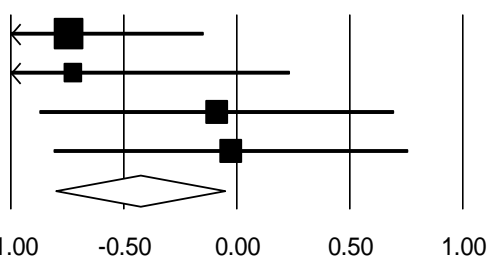

Visceral fat: MRI

Figure 2. Exercise intervention effects on overweight and obese adults 


\begin{tabular}{|c|c|c|c|c|}
\hline $\begin{array}{l}\text { First author (ye } \\
\text { ar), Country }\end{array}$ & Design & Participants & Exercise intervention & Major Findings \\
\hline $\begin{array}{l}\text { Batrakoulis, } \\
\text { (2018), Greece }\end{array}$ & $\begin{array}{l}\text { RCT: control }(n=21) \text {, exercise }(n=14) \text {, } \\
\text { exercise-detraining }(n=14)\end{array}$ & $\begin{array}{l}\text { Overweight or obese }(25.1-34.9 \mathrm{~kg} / \\
\left.\mathrm{m}^{2}\right) \text {, average } 36 \text { years old }\end{array}$ & $\begin{array}{l}40 \text { weeks, } 3 \text { times/week, combined exercises including } \\
\text { aerobic exercise, resistance exercise, and neuromotor } \\
\text { exercise, moderate intensity }\end{array}$ & $\begin{array}{l}\text { A whole-body dual-energy X-ray absorptiometry } \\
\text { scanner }\end{array}$ \\
\hline $\begin{array}{l}\text { Besnier (2015), } \\
\text { U.S.A. }\end{array}$ & $\begin{array}{l}\text { RCT: moderate intensity }(n=46) \text {, re } \\
\text { sistance training }(n=46), 60 \% \text { aero } \\
\text { bic exercise }(n=45) \text {, home exercise } \\
(45)\end{array}$ & $\begin{array}{l}\text { Obese older adults }(27-40 \mathrm{~kg} / \mathrm{m} \\
\left.{ }^{2}\right) \text {, average } 20-40 \text { years old }\end{array}$ & $\begin{array}{l}\text { RT: moderate intensity (cycle-ergometers, } 60 \% \mathrm{VO}_{2} \\
\max , 4 \text { days/week, } 55 \text { minutes) }\end{array}$ & Dual X-ray absorptiometry (DXA) \\
\hline $\begin{array}{l}\text { Blue (2018), } \\
\text { U.S.A. }\end{array}$ & $\begin{array}{l}\text { RCT: short interval training }(\mathrm{n}=1 \\
\text { 8), long interval training }(\mathrm{n}=16) \text {, a } \\
\text { nd control }(\mathrm{n}=9)\end{array}$ & $\begin{array}{l}\text { Obese adults }\left(25-45 \mathrm{~kg} / \mathrm{m}^{2}\right), 18- \\
50 \text { years old }\end{array}$ & $\begin{array}{l}8 \text { weeks, short interval training ( } 10 \text { repetition of } 1- \\
\text { minute bouts, } 90 \% \text { peak power output), long interv } \\
\text { al training ( } 5 \text { repetition of } 2 \text {-minute bouts, } 80-100 \% \\
\text { peak power output) }\end{array}$ & $\begin{array}{l}\text { Muscle cross-sectional area and thigh fat thicknes } \\
\text { s (ultrasound), lean mass and fat mass of legs } \\
\text { (DXA) }\end{array}$ \\
\hline $\begin{array}{l}\text { Brochu (2009), } \\
\text { Canada }\end{array}$ & $\begin{array}{l}\text { RCT: caloric restriction }(n=71) \text { vs. } \\
\text { caloric restriction and resistance ex } \\
\text { ercise }(n=36)\end{array}$ & $\begin{array}{l}\text { Overweight }\left(>27 \mathrm{~kg} / \mathrm{m}^{2}\right) \text {, averag } \\
\text { e } 58 \text { years old }\end{array}$ & $\begin{array}{l}6 \text { months, resistance exercise (phase } 1: 3 \text { weeks, } 15 \\
\text { repetitions or } \sim 65 \% \text { of maximum, and } 2 \sim 3 \text { sets, p } \\
\text { hase } 2: 5 \text { weeks, } 12 \text { repetitions or } \sim 70 \% \text { of maxim } \\
\text { um, and } 2 \sim 3 \text { sets, phase } 3: 9 \text { weeks, } 8 \sim 10 \text { repetitio } \\
\text { ns or } \sim 75 \%-80 \% \text { of maximum, and } 2 \sim 4 \text { sets and p } \\
\text { hase } 4: 8 \text { weeks, } 10 \sim 12 \text { repetitions, } \sim 70-75 \% \text { of } \\
\text { maximum, 3 4 sets) }\end{array}$ & Visceral fat (CT), abdominal fat area (CT) \\
\hline $\begin{array}{l}\text { Coker (2009), } \\
\text { UK }\end{array}$ & $\begin{array}{l}\text { RCT: moderate intensity exercise } \\
(\mathrm{n}=6) \text {, high intensity exercise }(\mathrm{n}= \\
6), \text { and control }(\mathrm{n}=6)\end{array}$ & $\begin{array}{l}\text { Overweight or obese }(26 \leq \mathrm{BMI} \\
\left.<37 \mathrm{~kg} / \mathrm{m}^{2}\right), 65-90 \text { years old }\end{array}$ & $\begin{array}{l}12 \text { weeks, } 1000 \text { kcal energy expend: cycle-ergomete } \\
\text { rs, } 50 \% \text { of } \mathrm{VO}_{2 \text { peak }} \text {, or } 75 \% \text { of } \mathrm{VO}_{2 \text { peak }}\end{array}$ & $\begin{array}{l}\text { Fat mass and lean tissue: X-ray, abdominal subcu } \\
\text { taneous adipose tissues and abdominal muscle wa } \\
\text { 11: CT }\end{array}$ \\
\hline $\begin{array}{l}\text { Gepner (2018), } \\
\text { U.S.A. }\end{array}$ & $\begin{array}{l}\text { RCT: exercise }(n=139) \text {, control }(n= \\
\text { 139) }\end{array}$ & $\begin{array}{l}\text { Overweight and obese older adult } \\
\mathrm{s}\left(27-41 \mathrm{~kg} / \mathrm{m}^{2}\right), \geq 55 \text { years old }\end{array}$ & $\begin{array}{l}18 \text { months, } 60 \text { minutes, } 65 \% \text { MHR of aerobic training, } \\
80 \% \text { of MHR of resistance training ( } 2 \text { sets, leg } \\
\text { extension, leg curl, elbow flexion, triceps extension, } \\
\text { lateral pull-down, lower back extension, bent leg sit- } \\
\text { ups) }\end{array}$ & $\begin{array}{l}\text { Visceral adipose tissue, intrahepatic fat, pancreatic } \\
\text { fat, intrapericardial fat, superficial subcutaneous } \\
\text { adipose tissue, deep subcutaneous adipose tissue, } \\
\text { renal sinus fat, and femur intermuscular adipose t } \\
\text { issue (MRI) }\end{array}$ \\
\hline $\begin{array}{l}\text { Goodpaster, }(2 \\
\text { 010), U.S.A. }\end{array}$ & $\begin{array}{l}\text { RCT: physical activity }(\mathrm{n}=67) \text {, and } \\
\text { control }(\mathrm{n}=63)\end{array}$ & $\begin{array}{l}\text { Obesity }\left(>30 \mathrm{~kg} / \mathrm{m}^{2}\right), 30-55 \text { years } \\
\text { old }\end{array}$ & $\begin{array}{l}12 \text { months, moderate intensity physical activity, bris } \\
\text { k walking, } 60 \text { minutes, } 5 \text { days/week, } 10,000 \text { steps/da } \\
\text { y }\end{array}$ & $\begin{array}{l}\text { Abdominal adipose tissues and hepatic fat content } \\
\text { s (CT) }\end{array}$ \\
\hline $\begin{array}{l}\text { Hunter (2010), } \\
\text { U.S.A. }\end{array}$ & $\begin{array}{l}\text { RCT: control }(n=26) \text {, aerobic exerc } \\
\text { ise }(n=15) \text {, resistance exercise }(n=1 \\
8)\end{array}$ & $\begin{array}{l}\text { Overweight women }(27 \leq \mathrm{BMI} \leq \\
\left.30 \mathrm{~kg} / \mathrm{m}^{2}\right), 21-46 \text { years old }\end{array}$ & $\begin{array}{l}1 \text { year, aerobic exercise (week 1: } 20 \text { minutes, } 67 \% \\
\text { maximum heart rate, and then continues duration a } \\
\text { nd intensity increased, week } 8: 80 \text { minutes, } 80 \% \text { of } \\
\text { maximum heart rate), resistance exercise (squats, } 1 \\
\text { eg extension, leg curl, elbow flexion, triceps extens } \\
\text { ion, lateral pull-down, bench press, military press, } 1 \\
\text { ower back extension, and bent leg sit-ups, } 10 \text { repeti } \\
\text { tions and } 80 \% \text { of } 1 \mathrm{RM} \text { ) }\end{array}$ & $\begin{array}{l}\text { Whole body lean and fat tissue (X-ray), intra- } \\
\text { abdominal adipose tissue, deep subcutaneous adipose } \\
\text { tissue, subcutaneous adipose tissue (CT) }\end{array}$ \\
\hline $\begin{array}{l}\text { Ibanez (2010), } \\
\text { Spain }\end{array}$ & $\begin{array}{l}\text { RCT: diet }(n=12) \text {, diet }+ \text { resistance } \\
\text { exercise }(n=13) \text {, and control (stret } \\
\text { ching, } n=9)\end{array}$ & $\begin{array}{l}\text { Obese women }\left(30-40 \mathrm{~kg} / \mathrm{m}^{2}\right) \text {, aver } \\
\text { age } 40-60 \text { years old }\end{array}$ & $\begin{array}{l}16 \text { weeks, leg extensor muscles, arm extensor musc } \\
\text { le, } 4-5 \text { muscle group exercise, } 70-80 \% \text { of } 1 \mathrm{RM}\end{array}$ & $\begin{array}{l}\text { Volumes of visceral and subcutaneous adipose tis } \\
\text { sue and muscle volume: MRI }\end{array}$ \\
\hline $\begin{array}{l}\text { Idoate (2011), } \\
\text { Spain }\end{array}$ & $\begin{array}{l}\text { RCT: diet }(n=12) \text {, diet }+ \text { resistance } \\
\text { exercise }(n=13) \text {, and control }(n=9)\end{array}$ & $\begin{array}{l}\text { Obesity }\left(30-40 \mathrm{~kg} / \mathrm{m}^{2}\right), 40-60 \text { year } \\
\text { s old }\end{array}$ & $\begin{array}{l}16 \text { weeks, } 50-70 \% 1 \mathrm{RM} \text {, leg extensor, bench press, } \\
\text { cardiovascular and whole-body conditioning exercis } \\
\text { e, } 20 \sim 60 \mathrm{~min}, 3 \text { time/week, }\end{array}$ & Abdominal adipose tissue (CT), SAT (MRI) \\
\hline $\begin{array}{l}\text { Irving (2008), } \\
\text { U.S.A. }\end{array}$ & $\begin{array}{l}\text { RCT: control }(n=7) \text {, low intensity } \\
\text { exercise }(n=11) \text {, and high intensity }\end{array}$ & $\begin{array}{l}\text { Obese women, average } 51 \text { years } \\
\text { old }\end{array}$ & $\begin{array}{l}16 \text { weeks, walking/running, low intensity RPE } \sim 10- \\
12 \text {, week } 1-2 \text { (300 kcal, } 1-2 \text { days/week), week } 3-4\end{array}$ & $\begin{array}{l}\text { Body fat, fat free mass, fat mass, abdominal fat, } \\
\text { subcutaneous fat, abdominal visceral fat, mid-thigh }\end{array}$ \\
\hline
\end{tabular}




\begin{tabular}{|c|c|c|c|c|}
\hline & exercise $(n=9)$ & & $\begin{array}{l}(350 \mathrm{kcal}, 4 \text { days/week), week 5-16 (400 kcal, } 5-1 \\
6 \text { days/week), high intensity RPE } \sim 15-17\end{array}$ & fat area, mid-thigh skeletal muscle: $\mathrm{CT}$ \\
\hline $\begin{array}{l}\text { Irwin (2003), } \\
\text { U.S.A. }\end{array}$ & $\begin{array}{l}\text { RCT: aerobic and resistance exerci } \\
\text { se ( } \mathrm{n}=87 \text { ), and control (stretching, } \\
\mathrm{n}=86 \text { ) }\end{array}$ & $\begin{array}{l}\text { Overweight or obese postmenopa } \\
\text { usal women }\left(\geq 35 \mathrm{~kg} / \mathrm{m}^{2}\right), 50-75 \\
\text { years old }\end{array}$ & $\begin{array}{l}7 \text { weeks, aerobic exercise }(60-75 \% \text { MHR, } 45 \text { minut } \\
\text { es), resistance exercise ( } 10 \text { repetitions } / 2 \text { sets, leg ext } \\
\text { ension, leg curls, leg press, chest press, and seated } \\
\text { dumbbell row) }\end{array}$ & $\begin{array}{l}\text { Total body fat, intra-abdominal fat, subcutaneous } \\
\text { abdominal fat (CT) }\end{array}$ \\
\hline $\begin{array}{l}\text { Janssen (2002), } \\
\text { Canada }\end{array}$ & $\begin{array}{l}\text { RCT: diet }(n=13), \text { diet }+ \text { aerobic e } \\
\text { xercise }(n=11) \text {, diet }+ \text { resistance ex } \\
\text { ercise }(n=14)\end{array}$ & $\begin{array}{l}\text { Obese women }\left(>27 \mathrm{~kg} / \mathrm{m}^{2}\right) \text {, avera } \\
\text { ge } 37 \text { years old }\end{array}$ & $\begin{array}{l}16 \text { weeks, aerobic exercise }(50-85 \% \text { MHR, } 60 \mathrm{minu} \\
\text { tes, } 5 \text { days/week), resistance exercise (leg extension, } \\
\text { leg flexion, super pullover, bench press, shoulder } \mathrm{pr} \\
\text { ess, triceps extension, biceps curl), } 8-12 \text { repetitions, } \\
3 \text { days/week }\end{array}$ & $\begin{array}{l}\text { Total fat, abdominal subcutaneous fat at L4-L5, s } \\
\text { keletal muscle, intramuscular fat: MRI }\end{array}$ \\
\hline $\begin{array}{l}\text { Johnson (200 } \\
9) \text {, Australia }\end{array}$ & $\begin{array}{l}\text { RCT: control }(n=8) \text {, and exercise } \\
(\mathrm{n}=12)\end{array}$ & Obesity $\left(\geq 35 \mathrm{~kg} / \mathrm{m}^{2}\right)$ & $\begin{array}{l}4 \text { weeks, a supervised, progressive aerobic exercise, } \\
\text { cycle ergometer, total } 30 \sim 34 \text { minutes ( } 15 \text { minutes s } \\
\text { essions and } 5 \text { minutes rest), } 3 \text { times/week, } 50 \% \mathrm{~V} \\
\text { O2peak for week } 1,60 \% \text { for week } 2 \text {, and } 70 \% \text { for } \\
\text { week } 3 \text { and } 4,15 \text { minutes sessions and } 5 \text { minutes } \\
\text { rest }\end{array}$ & $\begin{array}{l}\text { Hepatic triglyceride concentration and vastus later } \\
\text { alis intramyocellular triglyceride concentration (poi } \\
\text { nt-resolved spectroscopy), subcutaneous adipose ti } \\
\text { ssues area, hepatic lipid saturation index (HMRS), } \\
\text { visceral adipose tissue area (MRI) }\end{array}$ \\
\hline $\begin{array}{l}\text { Ko }(2016) \text {, } \\
\text { Canada }\end{array}$ & $\begin{array}{l}\text { RCT: combined exercise }(n=59), \text { a } \\
\text { nd control }(n=21)\end{array}$ & $\begin{array}{l}\text { Obese old adults, } 60-80 \text { years ol } \\
\text { d }\end{array}$ & $\begin{array}{l}6 \text { months, aerobic exercise (treadmill, } 5 \text { days/week, } \\
60-70 \% \mathrm{VO}_{2 \text { peak }}, 30 \text { minutes, resistance exercise }(3 \\
\text { days/week, chest press, shoulder raise, shoulder flex } \\
\text { ion, leg extension, biceps curl, abdominal crunches, } \\
\text { modified push-ups) }\end{array}$ & $\begin{array}{l}\text { Total, abdominal, abdominal subcutaneous, and vi } \\
\text { sceral adipose tissue (MRI) above the L4-5 interv } \\
\text { ertebral space }\end{array}$ \\
\hline $\begin{array}{l}\text { Larson-Meyer, } \\
\text { (2006), U.S.A. }\end{array}$ & $\begin{array}{l}\text { RCT: calorie restriction }(n=12) \text {, cal } \\
\text { orie resistance exercise }(n=12) \text {, low } \\
\text { calorie }(n=11) \text {, and control }(n=11)\end{array}$ & $\begin{array}{l}\text { Overweight }(25 \leq \text { BMI } \leq 30 \mathrm{~kg} \\
/ \mathrm{m} \\
\left.{ }^{2}\right) \text {, Caucasians, } 15 \text { African America } \\
\text { ns, and } 1 \text { Asian, } 25 \sim 50 \text { years old } \\
\text { for man, 25 45 years old for wo } \\
\text { men }\end{array}$ & $\begin{array}{l}6 \text { months, structured exercise (walking, running, or } \\
\text { stationary cycling), } 5 \text { days/week }\end{array}$ & $\begin{array}{l}\text { Visceral adipose tissue (CT), adipose tissue (X-ra } \\
\text { y), fat cell size (Multisizer-3 counter), ectopic fat } \\
\text { deposition in liver and muscle (point-resolved spe } \\
\text { ctroscopy) }\end{array}$ \\
\hline $\begin{array}{l}\text { Nicklas (2009), } \\
\text { U.S.A. }\end{array}$ & $\begin{array}{l}\text { RCT: caloric restriction }(n=29) \text {, cal } \\
\text { oric restriction and moderate intens } \\
\text { ity aerobic exercise }(n=36) \text {, caloric } \\
\text { restriction and vigorous intensity e } \\
\text { xercise }(n=30)\end{array}$ & $\begin{array}{l}\text { Overweight and obese postmenop } \\
\text { ausal women }(25 \leq \mathrm{BMI} \leq 40 \mathrm{k} \\
\left.\mathrm{g} / \mathrm{m}^{2}\right) \text {, average } 58 \text { years old }\end{array}$ & $\begin{array}{l}20 \text { weeks, treadmill at intensity of } 45-50 \% \text { (moderate } \\
\text { intensity) or } 70-75 \% \text { (vigorous intensity) of heart rate } \\
\text { reserve }\end{array}$ & $\begin{array}{l}\text { Whole body fat mass, lean mass, and percentage body } \\
\text { fat (X-ray), visceral and subcutaneous adipose tissue } \\
\text { volumes around abdomen (CT) }\end{array}$ \\
\hline $\begin{array}{l}\text { Park (2003), R } \\
\text { epublic of Kor } \\
\text { ea }\end{array}$ & $\begin{array}{l}\text { RCT: aerobic training group }(\mathrm{n}=1 \\
0) \text {, combined training group }(\mathrm{n}=1 \\
0) \text {, control }(\mathrm{n}=10)\end{array}$ & $\begin{array}{l}\text { Overweight or obese }(25-35 \mathrm{~kg} / \mathrm{m} \\
\left.{ }^{2}\right) \text {, average } 40 \text { years old }\end{array}$ & $\begin{array}{l}\text { Aerobic training }(60-70 \% \text { HRmax, } 60 \text { minutes, } 6 \\
\text { days/week), combined training groups ( } 3 \text { days/week } \\
\text { for resistance exercise, } 3 \text { days/week for aerobic exe } \\
\text { rcise) }\end{array}$ & $\begin{array}{l}\text { Abdominal visceral fat, subcutaneous fat and visc } \\
\text { eral fat (CT) }\end{array}$ \\
\hline $\begin{array}{l}\text { Park (2015), } \\
\text { Republic of } \\
\text { Korea }\end{array}$ & $\begin{array}{l}\text { RCT: combined exercise }(n=10), a \\
\text { nd control }(n=10)\end{array}$ & $\begin{array}{l}\text { Abdominal obese postmenopausal } \\
\text { women }\left(\geq 24 \mathrm{~kg} / \mathrm{m}^{2}\right) \text {, average } 57 \\
\text { years old }\end{array}$ & $\begin{array}{l}12 \text { weeks, resistance exercise ( } 70 \% \text { of } 1 \mathrm{RM}, 10-12 \\
\text { repetitions, } 3 \text { days/week, } 30 \text { minutes), aerobic exerc } \\
\text { ise ( } 40-75 \% \text { HRR, } 40 \text { minutes, } 3 \text { days/week) }\end{array}$ & Visceral fat $(\mathrm{CT})$ \\
\hline $\begin{array}{l}\text { Christiansen } \\
\text { (2009), } \\
\text { Denmark }\end{array}$ & $\begin{array}{l}\text { RCT: exercise }(n=25) \text {, hypocaloric } \\
\text { diet }(n=29) \text {, hypocaloric diet }+ \text { exe } \\
\text { rcise }(n=29)\end{array}$ & $\begin{array}{l}\text { Obese women }\left(30-40 \mathrm{~kg} / \mathrm{m}^{2}\right), 18- \\
45 \text { years old }\end{array}$ & $\begin{array}{l}12 \text { weeks, } 3 \text { times/week, } 60-75 \text { minutes, aerobic ex } \\
\text { ercise, } 70 \% \text { HRR, 500-600 kcal energy expenditure }\end{array}$ & Adipose tissue, skeletal muscle: MRI \\
\hline $\begin{array}{l}\text { Quist (2018), } \\
\text { Denmark }\end{array}$ & $\begin{array}{l}\text { RCT: aerobic exercise }(n=21) \text {, leis } \\
\text { ure-time exercise }(n=21) \text {, vigorous } \\
\text { intensity }(n=33) \text {, control }(n=16)\end{array}$ & $\begin{array}{l}\text { Obese adults }\left(25-35 \mathrm{~kg} / \mathrm{m}^{2}\right), 20- \\
45 \text { years old }\end{array}$ & $\begin{array}{l}6 \text { months, bike exercise }(320 \mathrm{kcal} / \mathrm{day} \text { for women, } \\
42 \mathrm{kcal} / \mathrm{day} \text { for men), leisure-time exercise of mode } \\
\text { rate }\left(50-70 \% \mathrm{VO}_{2 \text { peak }}\right) \text {, vigorous intensity }(50-70 \% \\
\left.\mathrm{VO}_{2 \text { peak }}\right)\end{array}$ & Body composition (DXA) \\
\hline Ross (2004), C & RCT: control $(\mathrm{n}=23)$, diet weight 1 & Overweight or obese $(>27 \mathrm{~kg} / \mathrm{m}$ & 14 weeks, $500 \mathrm{kcal}$ energy expend, daily exercise & Adipose tissue, skeletal muscle, fat-free skeletal \\
\hline
\end{tabular}




\begin{tabular}{|c|c|c|c|c|}
\hline anada & $\begin{array}{l}\text { oss }(n=28) \text {, exercise weight loss }(n \\
=23) \text {, exercise without weight loss } \\
(n=28)\end{array}$ & ${ }^{2}$ ), average 43 years old & (brisk walking or light jogging), $\sim 80 \%$ MHR & muscle \\
\hline $\begin{array}{l}\text { Schmitz }(200 \\
\text { 7), U.S.A. }\end{array}$ & $\begin{array}{l}\text { RCT: strength training }(\mathrm{n}=71 \text { at ye } \\
\text { ar } 1, \mathrm{n}=70 \text { at year } 2) \text { and control } \\
(\mathrm{n}=67 \text { at year } 1, \mathrm{n}=63 \text { at year } 3)\end{array}$ & $\begin{array}{l}\text { Overweight or obese }(25-35 \mathrm{~kg} / \mathrm{m} \\
\left.{ }^{2}\right), 25-44 \text { years old }\end{array}$ & $\begin{array}{l}16 \text { weeks, } 2 \text { days/week, } 3 \text { sets of } 8-10 \text { repetitions, } \\
\text { quadriceps, hamstring, gluteal, pectoral, erector spinae, } \\
\text { latissimus dorsi, rhomboid, deltoid, biceps, and triceps } \\
\text { muscles }\end{array}$ & $\begin{array}{l}\text { Body composition: DAX, abdominal fat areas (tot } \\
\text { al, subcutaneous, and intraabdominal): CT at the } \\
\text { L2-L3 interspace }\end{array}$ \\
\hline $\begin{array}{l}\text { Slentz (2005), } \\
\text { U.S.A. }\end{array}$ & $\begin{array}{l}\text { RCT: high amount/vigorous intensity } \\
(\mathrm{n}=42) \text {, low amount/vigorous } \\
\text { intensity }(\mathrm{n}=46), \text { low } \\
\text { amount/moderate intensity }(\mathrm{n}=40) \text {, } \\
\text { and control }(\mathrm{n}=47)\end{array}$ & $\begin{array}{l}\text { Overweight and obese }(25 \leq \mathrm{BMI} \\
\left.\leq 35 \mathrm{~kg} / \mathrm{m}^{2}\right), 137 \text { Caucasians, } 2 \\
9 \text { African Americans, } 49 \text { Asians/ } \\
\text { Hispanics, } 40 \sim 65 \text { years old }\end{array}$ & $\begin{array}{l}8 \text { months, 1) high amount/vigorous intensity (joggin } \\
\text { g } 20 \text { miles/week), 2) low amount/vigorous intensity } \\
\text { (jogging } 12 \mathrm{miles} / \text { week), and } 3 \text { ) low amount/moder } \\
\text { ate intensity (walking } 12 \mathrm{miles} / \text { week) }\end{array}$ & $\begin{array}{l}\text { Visceral fat (CT), Subcutaneous fat, Total abdomi } \\
\text { nal fat, body weight }\end{array}$ \\
\hline $\begin{array}{l}\text { Thong (2000), } \\
\text { Canada }\end{array}$ & $\begin{array}{l}\text { RCT: control }(\mathrm{n}=8) \text {, diet }+ \text { weight } \\
\text { loss }(\mathrm{n}=14) \text {, exercise }+ \text { weight loss } \\
(\mathrm{n}=14) \text {, and exercise }+ \text { weight ma } \\
\text { intain }(\mathrm{n}=16)\end{array}$ & $\begin{array}{l}\text { Obese men }\left(\geq 30 \mathrm{~kg} / \mathrm{m}^{2}\right) \text {, average } \\
44 \text { years old }\end{array}$ & $\begin{array}{l}12 \text { weeks, } 700 \mathrm{kcal} \text { energy expend: } 75 \% \mathrm{VO}_{2 \max }(\sim \\
80 \% \text { MHR), walking or jogging }\end{array}$ & $\begin{array}{l}\text { Total adipose tissue, subcutaneous adipose tissue, } \\
\text { visceral adipose tissue: MRI }\end{array}$ \\
\hline $\begin{array}{l}\text { Verreijen }(20 \\
17), \text { Netherland } \\
\mathrm{s}\end{array}$ & $\begin{array}{l}\text { RCT: control }(n=14) \text {, protein }(n=1 \\
3) \text {, resistance exercise }(n=19) \text {, and } \\
\text { protein and resistance exercise }(n= \\
\text { 22) }\end{array}$ & $\begin{array}{l}\text { Obese older adults (average } 32.2 \\
\mathrm{~kg} / \mathrm{m}^{2} \text { ), average } 62.4 \text { years old }\end{array}$ & $\begin{array}{l}10 \text { weeks, resistance training ( } 3 \text { days/week, 1hour, } \\
\text { squats, lunges, chest press, shoulder press, biceps c } \\
\text { urls, triceps extensions, standing rows, step-ups, cru } \\
\text { nches) }\end{array}$ & $\begin{array}{l}\text { Body composition (air displacement } \\
\text { plethysmography) }\end{array}$ \\
\hline
\end{tabular}

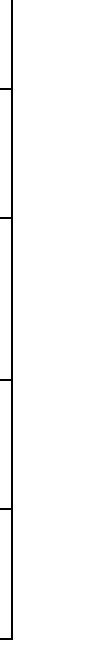

.

quadriceps, hamstring, gluteal, pectoral, erector spi

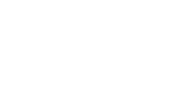




\section{Discussion}

Authors should discuss the results and how they can be interpreted in perspective of previous studies and of the working hypotheses. The findings and their implications should be discussed in the broadest context possible. Future research directions may also be highlighted. This meta-analysis found that participating in exercise interventions had a positive influence on reducing weight and subcutaneous, visceral, and ectopic fat in overweight or obese individuals. Only a limited number of studies regarding the effects of exercise intervention on overweight or obese adults are available for review. Studies with ectopic fat measurements are particularly rare. We conducted a meta-analysis to find the effects of exercise intervention on overweight or obese adults as measured by accumulation of fat.

Exercise intervention had a beneficial effect on reducing weight and fat accumulation, including both subcutaneous and ectopic fat, among overweight or obese adults. Fat distributions accumulate as subcutaneous, visceral, and ectopic fat in other organs depending on their capacities for fat storage [31]. A recent meta-analysis of three studies reported that overweight or obese children and adolescents had beneficial effects from exercise intervention. These children and adolescents had reductions in subcutaneous, visceral, and ectopic fat accumulation after completing exercise interventions [7]. However, no meta-analysis study focusing on adults has been performed. This metaanalysis selected all studies that reported effects of exercise interventions on reducing weight and fat accumulation in overweight or obese adults. We found degrees of effectiveness on subcutaneous and visceral fat, but we were unable to ascertain the same effectiveness on ectopic fat due to the limited number of studies in which ectopic fat was measured. This should be addressed in future studies as a recent meta-analysis of three studies, two of which were conducted in the same laboratory, found that hepatic fat in obese children and adolescents who participated in exercise interventions was decreased.

While the exercise interventions were effective in reducing the accumulated fat in overweight or obese adults, the degrees of effectiveness varied according to method of measurement, either CT or MRI. CT and MRI were used to measure subcutaneous and visceral fat in all selected studies. Visceral fat as measured by CT demonstrated a substantial effect with large heterogeneity while subcutaneous fat measured by CT and visceral fat measure by MRI demonstrated less effectiveness and smaller heterogeneity. Subcutaneous fat reduction measured by MRI was not significantly effective due to a small sample size and large heterogeneity. Also, we were unable to compute the effectiveness of exercise intervention on ectopic fat deposition; no MRI studies on liver adipose tissues had been conducted in overweight or obese adults. Considering the differences in measurement methods, further studies are needed to delineate clinically meaningful measurements of accumulated fat. Exercise interventions consisted of moderate to vigorous intensity, 50 minutes per session, and 4 times per week for 22 weeks. Exercise types included aerobic, resistance, combined aerobic and resistance, and interval training. These are important for designing an evidence-based exercise intervention for overweight or obese adults. Current findings of exercise intensity and durations were higher than recommendations of the American College of Sports Medicine (ACSM) for long-term weight loss, which is 200-300 minutes per week of moderate intensity exercise. Maintaining weight loss and preventing weight regain after completing exercise interventions need to be addressed in future studies through long-term follow-up.

There are four potential mechanisms for the findings of this study. First, exercise helps to increase skeletal muscle including increased muscle mass and strength, skeletal muscle glucose uptake, and fatty acid oxidation [32]. Second, the effects of exercise on liver tissues may include increased hepatic uptake of fatty acids and decreased hepatic glucose production, cholesterol synthesis, and glycogen synthesis [33]. Third, in adipose tissue, exercise works to reduce fat mass and leptin and resistin production and increase lipolysis and adiponectin production [34]. Last, participating in exercise decreased chronic inflammation and increased growth factor production leading to endocrine changes that improve systemic mechanisms. 
This study had some limitations. First, selected studies were still too limited for generalizing to all overweight or obese adults. Second, while reliability and validity were found, all measurements of each organ's adipose tissues were different in the selected study. Third, we suggested a guideline for exercise interventions in overweight or obese individuals based on descriptions of the selected studies, but the guideline requires improvement in detail. Last, this meta-analysis included all races, but an exercise guideline should be developed considering differences in race.

\section{Conclusions}

Overweight or obese adults who participated in exercise interventions demonstrated weight and subcutaneous and visceral fat reduction. All types of exercise including aerobic and resistance exercise were effective; and moderate to vigorous intensity exercise programs, 4 times per week, for 50 minutes, and 22 weeks duration may be the most effective based on the available evidence.

Author Contributions: Conceptualization, J.L.; methodology, J.L.; software, J.L.; validation, J.L.; formal analysis, J.L.; investigation, J.L.; resources, J.L.; data curation, J.L.; writing-original draft preparation, J.L.; writing - review and editing, J.L.; visualization, J.L.; supervision, J.L.; project administration, J.L.; funding acquisition, J.L. All authors have read and agreed to the published version of the manuscript.

Funding: This research received no external funding.

Acknowledgments: None

Conflicts of Interest: The authors declare no conflict of interest.

References

1. WHO. Obesity and overweight. Geneva, Switzerland, 2018; pp https:/www.who.int/newsroom/fact-sheets/detail/obesity-and-overweight.

2. Flegal, K.M.; Kit, B.K.; Orpana, H.; Graubard, B.I. Association of all-cause mortality with overweight and obesity using standard body mass index eategories: a systematic review and meta-analysis. JAMA 2013, 309, 71 -82, doi:1 0.1001/jama.201 2.113905.

3. Lim, S.; Meigs, J.B. Eetopic fat and cardiometabolic and vascular risk. Int J Gardiol 2013,169, 166-1 76, doi: 10.1016/j.ijeard.2013.08.077.

4. Gastaldelli, A.; Basta, G. Eetopic fat and eardiovaseular disease: what is the link? Mutr Metab Cardiovase Dis 2010, 20, 481-490, doi: $10.1016 / j$.numeed.2010.05.005.

5. Siegel-Axel, D.lo; Haring, H.U. Perivaseular adipose tissue: An unique fat compartment relevant for the cardiometabolic syndrome. Rer Endoer Metab Disord 2016, 17, 51 -60, doi: $10.1007 / s 11$ 154-016-9346-3.

6. Lim, S. Ectopic fat assessment focusing on cardiometabolic and renal risk. Endocrinol Metab (Seoul) 2014, 29, 1-4, doi:10.3803/EnM.2014.29.1.1.

7. Hens, Wo; Vissers, D.; Hansen, D.; Peeters, S.; Gielen, J.; Van Gaal, L.; Taeymans, J. The effect of diet or exereise on ectopic adiposity in children and adolescents with obesity: a systematic review and meta-analysis. Obes Rev 2017, , $8,1310-1322$, doi: 10.111 I/obr. 12571.

8. Liberati, A.; Altman, D.Go; Tetzlaff, J.; Mulrow, G.; Gotzsche, P.G.; Ioannidis, J.P.; Glarke, Mo; Devereaux, P.J.; Kleijnen, J.; Moher, D. The PRISMA statement for reporting systematic reviews and meta-analyses of studies that evaluate health care interventions: explanation and elaboration. Ann Intern Med 2009, 15/, W65-94, doi:10.7326/0003-4819-1 51-4-200908 180. 00136.

9. Higgins, J.P.; Altman, D.Go; Gotzsehe, P.G.; Juni, Po; Moher, D.; Oxman, A.D.; Savovie, J.; Sehulz, K.F.; Weeks, L.; Sterne, J.A., et al. The Gochrane Collaboration's tool for assessing risk of bias in randomised trials. BMJ 2011 , 343, d5928, doi: 10.11 36/bmj.d5928.

10. Larson-Meyer, D.E.; Heilbronn, LoK.; Redman, L.Mo; Newcomer, B.R.; Frisard, M.lo; Anton, S.; Smith, S.R.; Alfonso, A.; Ravussin, E. Effeet of calorie restriction with or without exereise on insulin sensitivity, beta-cell function, fat cell size, and ectopic lipid in overweight subjects. 
Diabetes Care 2006, 29, $1337-1$ 344, doi:10.2337/de05-2565.

II. Slentz, G.A.; Aiken, L.B.; Houmard, J.A.; Bales, C.W.; Johnson, J.Lo; Tanner, G.J.; Duseha, B.D.; Kraus, W.E. Inactivity, exereise, and viseeral fat. STRRIDE: a randomized, controlled study of exereise intensity and amount. J Appl Physiol (1985) 2005, 99, 1613-1618, doi: 10.1152 /japplphysiol.001 24.2005.

1 2. Johnson, M.A.; Sachinwalla, To; Walton, D.W.; Smith, K.; Armstrong, A.; Thompson, M.W.; George, J. Aerobic exercise training reduces hepatic and visceral lipids in obese individuals without weight loss. Hepatology 2009, 50, 11 105-1 11 2, doi:10.1 002/hep. 23129.

13. Brochu, M.; Malita, M.F.; Messier, Vo; Doucet, E.; Strychar, I.; Lavoie, J.M.; Prud'homme, D.; Rabasa-Lhoret, Ro Resistance training does not contribute to improving the metabolic profile after a 6-month weight loss program in overweight and obese postmenopausal women. J Glin Endocrinol Metab 2009, 94, 3226-3233, doi:10.1 21 0/je.2008-2706.

14. Hunter, G.R.; Brock, D.W.; Byrne, M.M.; Chandler-Laney, P.G.; Del Gorral, P.; Gower, B.A. Exereise training prevents regain of viseeral fat for 1 year following weight loss. Obesity (Silver Spring) 2010, 18, 690-695, doi:10.1038/0by.2009.316.

15. Nieklas, B.J.; Wang, X.; You, To; Lyles, M.F.; Demons, J.; Easter, L.; Berry, M.J.; Lenchik, L.; Carr, J.J. Effeet of exereise intensity on abdominal fat loss during calorie restriction in overweight and obese postmenopausal women: a randomized, controlled trial. Am J Glin Wutr 2009, 89, 1043-1052, doi:1 10.3945/ajen.2008.26938.

16. Irving, B.A.; Davis, G.K.; Brock, D.W.; Weltman, J.Y.; Swift, D.; Barrett, E.J.; Gaesser, G.A.; Weltman, A. Effeet of exereise training intensity on abdominal visceral fat and body composition. Med Sei Sports Exere 2008, 40, 1863-1872, doi:1 0.1 249/MSS.0bo I 3e3181801 d40.

11. Thong, F.S.; Melean, C.; Graham, T.E. Plasma leptin in female athletes: relationship with body fat, reproductive, nutritional, and endoerine factors. J Appl Physiol (1985) 2000, 88, 2037. 2044, doi:10.1 152/jappl.2000.88.6.2037.

18. Ibanez, J.; Izquierdo, M.; Martinez-Labari, G.; Ortega, F.; Grijalba, A.; Forga, L.; Idoate, Fo; GareiaUneiti, M.; Fernandez-Real, J.M.; Gorostiaga, E.M. Resistance training improves cardiovaseular risk factors in obese women despite a significative decrease in serum adiponeetin levels. Obesity (Silver Spring) 2010, 18, 535-541, doi:10.1038/0by.2009.211.

19. Janssen, lo; Fortier, A.; Hudson, R.; Ross, R. Effeets of an energy-restrietive diet with or without exereise on abdominal fat, intermuseular fat, and metabolic risk factors in obese women. Diabetes Gare 2002, 25, 431-438, doi:10.2337/diacare.25.3.431.

20. Sehmitz, K.H.; Hannan, P.J.; Stovitz, S.D.; Bryan, C.J.; Warren, M.; Jensen, M.D. Strength training and adiposity in premenopausal women: strong, healthy, and empowered study, Am J Glin Wutr 2007, 86, 566-572, doi:10.1093/ajen/86.3.566.

21. Park, S.K.; Park, J.H.; Kwon, Y.G.; Kim, H.S.; Yoon, M.S.; Park, H.T. The effeet of combined aerobic and resistance exereise training on abdominal fat in obese middle-aged women. $J$ Physiol Anthropol Appl Human Sei 2003, 22, 1 $29-135$.

22. Ross, R.; Janssen, lo; Dawson, J.; Kungl, A.M.; Kuk, J.Lo; Wong, S.L.; Nguyen-Duy, T.B.; Lee, S.; Kilpatrick, K.; Hudson, R. Exereise-induced reduction in obesity and insulin resistance in women: a randomized controlled trial. Obes Res 2004, 12, 189-798, doi:10.1038/oby.2004.95.

23. Goodpaster, B.H.; Delany, J.P.; Otto, A.D.; Kuller, L.; Vockley, J.; South-Paul, J.E.; Thomas, S.B.; Brown, J.; MeTigue, K.; Hames, K.C., et al. Effects of diet and physieal aetivity interventions on weight loss and cardiometabolic risk factors in severely obese adults: a randomized trial. JAMA 2010, 304, 1795-1802, doi:10.1001/jama.2010.1505.

24. Idoate, F.; Ibanez, J.; Gorostiaga, E.M.; Gareia-Uneiti, M.; Martinez-Labari, G.; Izquierdo, M. Weight-loss diet alone or combined with resistance training induces different regional viseeral fat changes in obese women. Int J Obes (Lond) 201 I, 35, 700-7 I 3, doi:1 0.1038/ijo.2010.190.

25. Blue, M.M.M.; Smith-Ryan, A.E.; Trexler, E.To; Hirseh, K.R. The effeets of high intensity interval training on musele size and quality in overweight and obese adults. J Sei Med Sport 2018, 21 , 207-2 1 2, doi:10.1016/j.jsams.2011.06.001.

26. Quist, J.S.; Rosenkilde, M.; Petersen, M.B.; Gram, A.S.; Sjodin, A.; Stallknecht, B. Effeets of active commuting and leisure-time exereise on fat loss in women and men with overweight and obesity: a randomized controlled trial. Int J Obes (Lond) 2018, 42, 469-478, 
doi:10.1038/ijo.2017.253.

21. Besnier, F.; Lenelume, V.; Gerardin, P.; Fianu, A.; Martinez, J.; Naty, Mo; Poreherat, S.; Boussaid, K.; Sehneebeli, S.; Jarlet, E., et al. Individualized Exereise Training at Maximal Fat Oxidation Combined with Fruit and Vegetable-Rich Diet in Overweight or Obese Women: The LIPOXmaxReunion Randomized Controlled Trial, PLOS One 2015, 10, e0139246, doi:10.1 371 /journal.pone.0139246.

28. Batrakoulis, A.; Jamurtas, A.Z.; Georgakouli, K.; Draganidis, D.; Deli, C.K.; Papanikolaou, K.; Avloniti, A.; Chatzinikolaou, A.; Leontsini, D.; Tsimeas, P., et al. High intensity, eireuit-type integrated neuromuseular training alters energy balance and reduces body mass and fat in obese women: A 10-month training-detraining randomized controlled trial, PLOS One 2018, 13, e0202390, doi:10.1 37 1/journal.pone.0202390.

29. Park, S.M.; Kwak, Y.S.; Ji, J.G. The Effeets of Combined Exereise on Health-Related Fitness, Endotoxin, and Immune Funetion of Postmenopausal Women with Abdominal Obesity. $J$ Immunol Res 2015, 2015, 830567, doi:10.1 155/2015/830567.

30. Christiansen, To; Paulsen, S.K.; Bruun, J.M.; Overgaard, K.; Ringgaard, S.; Pedersen, S.B.; Positano, Vo; Richelsen, B. Comparable reduetion of the viseeral adipose tissue depot after a diet-induced weight loss with or without aerobic exereise in obese subjects: a 12 -week randomized intervention study, Eur J Endocrinol 2009, 160, 159-767, doi:10.1530/EJE-081009.

31. Zamboni, M.; Rossi, A.P.; Fantin, F.; Budui, S.L.; Zoico, E.; Zamboni, G.A.; Mazzali, G. Predietors of Eetopic Fat in Humans. Curr Obes Rep 2014, 3, 404-41 3, doi:1 0.1007/s 1 3679-014-01 26-1.

32. Pedersen, B.K.; Febbraio, M.A. Museles, exereise and obesity: skeletal musele as a secretory organ. Nat Rev Endocrinol 201 2, 8, 457-465, doi:10.1038/nrend0.201 2.49.

33. Zinker, B.A.; Mohr, T.; Kelly, P.; Namdaran, K.; Bracy, D.P.; Wasserman, D.H. Exereise-induced fall in insulin: mechanism of action at the liver and effects on muscle glucose metabolism. $\mathrm{Am} \mathrm{J}$ Physiol 1994, 266, E683-689, doi:10.1 152 /ajpendo.1994.266.5.E683.

34. Kramer, C.K.; Zinman, B.; Retnakaran, R. Are metabolically healthy overweight and obesity benign conditions?: A systematic review and meta-analysis, Ann Intern Med 201 3, 159, 158 169, doi:1 0.7326/0003-481 9-159-1 1-201 31 2030-00008. 